VARIABLE STARS

\section{Absorption in the Infrared}

\section{by our Cosmology Correspondent}

A CORRELATION between the infrared absorption band of $\mathrm{H}_{2} \mathrm{O}$ at $1.9 \mu \mathrm{m}$ and the microwave emission of the same molecule in long-period Mira variables suggests details of the mass loss mechanism operating in these stars.

J. A. Frogel reports that absorption features characteristic of water vapour have been detected in the spectra of seventeen Mira variables, and that the variation of the intensity of the $1.9 \mu \mathrm{m}$ band is inversely correlated with that of the radio emission of this molecule at $1.35 \mathrm{~cm}$ and with the optical luminosity variations (Astrophys. J., 162, L5; 1970). The infrared measurements have been carried out using a $0.5 \mathrm{~m}$ Ebcrt-Fastie spectrometer; Frogel used a comparison between the $1.9 \mu \mathrm{m}$ band and the $2.25 \mu \mathrm{m}$ band to determine the variation at $1.9 \mu \mathrm{m}$, because at $2.25 \mu \mathrm{m}$ there is neither stellar absorption nor terrestrial atmospheric absorption, so that measurements at this wavelength provide a good indication of the variation of the total light curve of the star. Relative to the $2.25 \mu \mathrm{m}$ feature, the $1.9 \mu \mathrm{m}$ absorption rises to a maximum just before the minimum of the light curve, after which it decreases rapidly to a minimum value which is maintained for roughly one-fifth of the optical period before a slow rise to the next maximum. The available data at microwave frequencies suggest that the emission at $1.35 \mathrm{~cm}$ is positively correlated with the optical variations (compare $\mathbf{P}$. Schwartz and $\mathrm{A}$. Barrett, Astrophys. J., 159, L123; 1970).

The model which Frogel proposes to explain this behariour depends in detail on the way in which mass loss occurs from these stars. He suggests that around the maximum of the optical intensity so much material is expelled above the photosphere that the water which forms (independently of pressure bolow 2,500 K) increases in quantity so rapidly that at first this gain outweighs the decrease in absorptivity resulting from its falling temperature. Once the star is well passed maximum intensity, the rapid cooling of the water vapour and lack of any new material soon produce a decrease in the observed absorption to a minimum. As the cycle repeats, the water vapour surrounding the star remains associated as $\mathrm{H}_{2} \mathrm{O}$, and just before maximum luminosity conditions are suitable for the pumping required to produce the observed microwave radiation by maser action. This model is very susceptible to observational tests, because it intimately relates the microwave and infrared behaviour of the sources. Conditions suitable for the formation of $\mathrm{H}_{2} \mathrm{O}$ might also be expected to be close to those needed for production of $\mathrm{OH}$, so that these three classes of emission or absorption objects could well be members of one basic family.

\section{SOLIDS}

\section{X-rays and Shock Waves}

Autrockir X-ray diffraction has been used to study the structure of solids since near the beginning of the twentieth century, it has usually been a technique carried out over fairly long periods of time with crystals which were not subject to sudden large distortions. The desire to look at solids undergoing

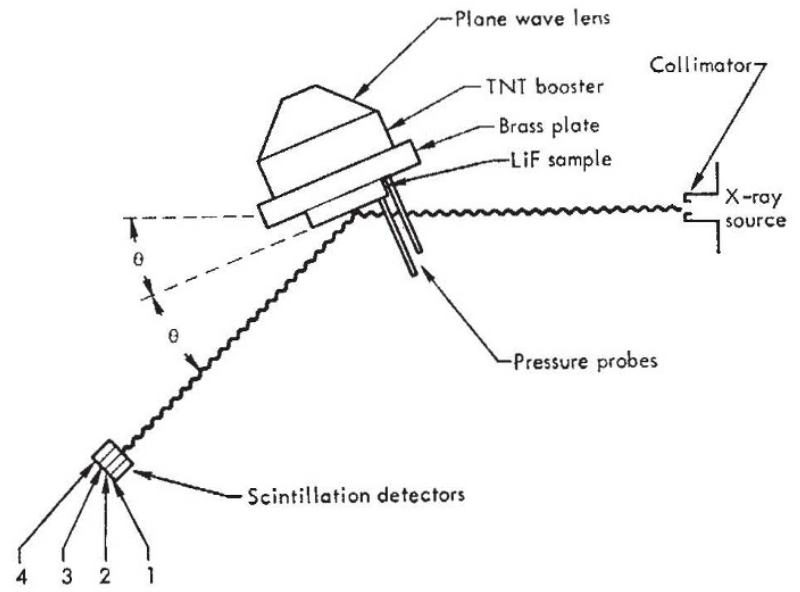

Channel numbers

Experimental arrangement for X-ray diffraction study of [LiF under shock compression.

rapid deformations has increased with the ever growing interest in the interaction of, for example, phonons and laser radiation with matter. The problems of looking at phenomena in solids which last only a few tens of nanoseconds are, however, great and are associated chiefly with the switching of a sufficiently short and intense pulse of X-rays at the correct time.

Johnson et al. report from the Lawrence Radiation Laboratory of the University of California (Physical Review Letters, 25, 1099; 1970) that they have devised an experimental system which has broadly overcome these problems. They do not describe their pulsed X-ray generator in detail, but the apparatus with which they have managed to observe $\mathrm{X}$-ray diffraction from a crystal of lithium fluoride under shock wave compression is illustrated here.

The transit time for the shock wave from a small TNT charge was measured with two pressure probes which defined the time of entry and exit of the shock wave and made it possible to calculate the shock pressure. Because most of the diffracted X-rays come from very close to the surface of the crystal, it is important to switch on the X-rays about $30 \mathrm{~ns}$ before the estimated time of breakout of the shock wave so that the compressed state can be properly observed. If X-rays were truly diffracted from the compressed state of the crystal, the signals from channel 2 would be expected to increase by a factor of about three and those from channel 4 to decrease by about the same amount. In the best experimental run, the output from channel 2 was found to be up by a factor of about $2 \cdot 7$ and that from channel 4 down by a factor of about $0 \cdot 7$. The centre of each scintillator is about $0.2 \mathrm{~cm}$ from its neighbour and the resolution of the apparatus is clearly not very high, but if it is assumed that a diffraction line shift from channel 4 to channel 2 has taken place, the corresponding $12 \theta\left(\sim 2 \cdot 6^{\circ}\right)$ is quite close to that expected for an isotropic applied pressure of the size calculated from the shock transit time $(\sim 130 \mathrm{kbar})$. In other words, the unit cell of the lattice is being compressed from a cube into a smaller cube rather than into a rectangular box of square cross-section, as might be intuitively expected when a plane shock wave is applied to a crystal. As well as establishing a technique which will doubtless be improved in the near future, this work secms to have thrown up some interesting side issues. 